\title{
Mielinólise pontina: relato de caso
}

\author{
Pontine Myelinolysis: Case report \\ Mielinólisis Pontina: Relato de caso
}

Camila Duque Fagundes de Figueiredo ${ }^{1}$, Marcos Vinicius Nunes Martins ${ }^{1}$, Américo Alves de Almeida Junior ${ }^{1,2,3}$, Evandro Barbosa dos Anjos ${ }^{1}$, Marcelo José da Silva de Magalhães ${ }^{1,2,3,4 *}$.

\section{RESUMO}

Introdução: A mielinólise pontina central (MPC) e a mielinólise extrapontina (MEP) são agrupadas dentro do contexto de síndromes de desmielinização osmótica as quais são caracterizadas pela dissolução das bainhas de mielina, preservando os axônios. O objetivo deste relato de caso é descrever o relato de caso de paciente com quadro de MPC e realizar a revisão na literatura médica. Detalhamento de caso: Trata-se de uma paciente do sexo feminino, 57 anos, que desenvolveu MPC após rápida correção da hiponatremia secundária a vômitos persistentes. Evolui com síndrome pseudobulbar, tetraparesia, disartria, rebaixamento do nível de consciência e disfagia. Após cinco meses de internação para tratamento sintomático e das complicações inerentes ao seu quadro clínico evoluiu com melhora parcial dos sintomas motores. Discussão: A etiologia da MPC e MEP é multifatorial, com incidência desconhecida e prevalência igual em ambos os sexos, podendo ser encontrada em todas as idades, com elevadas taxas de morbimortalidade. Embora seu principal fator desencadeante seja a correção excessivamente rápida da hiponatremia grave, alguns pacientes podem desenvolvê-la com concentrações séricas de sódio mais elevadas e com menores taxas de correção. 0 diagnóstico é essencialmente clínico, porém a ressonância magnética é o exame complementar de eleição para confirmação. Os pacientes podem apresentar desde recuperação completa até sequelas neurológicas irreversíveis, não existindo tratamento específico.

Palavras-chave: Mielinólise pontina, Hiponatremia, Tetraparesia.

\begin{abstract}
Introduction: Central pontine myelinolysis (CPM) and extrapontine myelinolysis (EPM) are grouped within the context of osmotic demyelination syndromes which are characterized by the dissolution of the myelin sheaths, preserving the axons. The objective of this case report is to describe the case report of a patient with CPM and review the medical literature. Details of case report: This is a 57-year-old female patient who developed CPM after rapid correction of hyponatremia secondary to persistent vomiting. It evolves with pseudobulbar syndrome, tetraparesis, dysarthria, lowering of consciousness level and dysphagia. After five months of hospitalization for symptomatic treatment and complications inherent to his clinical condition, he evolved with partial improvement of motor symptoms. Discussion: The etiology of CPM and EPM is multifactorial, with unknown incidence and equal prevalence in both sexes, and can be found at all ages, with high morbidity and mortality rates. Although its main triggering factor is the overly rapid correction of severe hyponatremia, some patients may develop it with higher serum sodium concentrations and lower correction rates. The diagnosis is essentially clinical, but magnetic resonance imaging is the complementary exam of choice for confirmation. Patients may present from complete recovery to irreversible neurological sequelae, and there is no specific treatment.
\end{abstract}

Keywords: Pontine myelinolysis, Hyponatremia, Tetraparesia.

\footnotetext{
${ }_{1}^{1}$ Faculdades Unidas do Norte de Minas (FUNORTE), Montes Claros-MG.

2 Faculdades Integradas Pitágoras (FipMOC), Montes Claros-MG

${ }^{3}$ Hospital Aroldo Tourinho, Montes Claros-MG.

${ }^{4}$ Hospital Vila da Serra-Nova Lima-MG. * E-mail: marcelo7779@yahoo.com.br
} 


\section{RESUMEN}

Introducción: La mielinólisis pontina central (MPC) y la mielinólisis extrapontina (MEP) se agrupan dentro del contexto de síndromes de desmielinización osmótica, que se caracterizan por la disolución de las vainas de mielina, preservando los axones. El objetivo de este relato de caso es describir el relato de caso de paciente con cuadro de MPC y realizar la revisión en la literatura médica. Detalle de caso: Se trata de una paciente del sexo femenino, de 57 años, que desarrolló MPC después de una rápida corrección de la hiponatremia secundaria a vómitos persistentes. Evoluciona con síndrome pseudobulbar, tetraparesia, disartria, descenso del nivel de conciencia y disfagia. Después de cinco meses de internación para tratamiento sintomático y de las complicaciones inherentes a su cuadro clínico evolucionó con mejoría parcial de los síntomas motores. Discusión: La etiología de la MPC y MEP es multifactorial, con incidencia desconocida y prevalencia igual en ambos sexos, pudiendo ser encontrada en todas las edades, con altas tasas de morbimortalidad. Aunque su principal factor desencadenante es la corrección excesivamente rápida de la hiponatremia grave, algunos pacientes pueden desarrollarla con concentraciones séricas de sodio más elevadas y con menores tasas de corrección. El diagnóstico es esencialmente clínico, pero la resonancia magnética es el examen complementario de elección para confirmación. Los pacientes pueden presentar desde recuperación completa hasta secuelas neurológicas irreversibles, no existiendo tratamiento específico.

Palabras clave: Mielinólisis pontina, Hiponatremia, Tetraparesia.

\section{INTRODUÇÃO}

Mielinólise pontina central (MPC) define-se como uma doença desmielinizante aguda e grave, não inflamatória, que atinge axônios localizados na região centro-pontina, podendo acometer em 10\% dos casos outras regiões como tálamo, globo pálido, putame, substância branca e cerebelo, nestes casos com a denominação mielinólise extrapontina (MEP). Dentre as principais manifestações do quadro temos: a paralisia pseudobulbar, tetraparesia espástica e alterações agudas do estado mental (BENTO e AUGUSTO, 2013; ADAMS et al.,1959).

Essa doença foi descrita em 1959, a partir da observação de um paciente etilista que apresentou quadro neurológico de tetraparesia associado à paralisia pseudobulbar. Ele havia sido internado para tratamento de do etilismo crônico e desenvolveu Delirium tremens. Após o seu óbito foi identificado a presença de lesão simétrica, de característica desmielinizante na base pontina (ADAMS et al.,1959). Na descrição desses autores, não havia referência a alterações hidroeletrolíticas que pudessem estar associadas ao quadro clínico. Somente em 1976, foi relacionado à MPC a alterações eletrolíticas (BROWN, 2000; ADAMS et al.,2009).

A incidência é desconhecida, mas numa série de 3.548 autópsias consecutivas em adultos, a lesão típica foi detectada em 9 casos $(0,25 \%)$. Sua prevalência é igual em ambos os sexos, e pode ser encontrada em todas as idades, apresentando elevada mortalidade e morbidade (BENTO et al, 2013; ADAMS et al.,2009).

Suspeita-se que a MPC possa apresentar uma etiologia que envolva múltiplos fatores. Esse processo fisiopatológico envolveria deficiências no metabolismo energético, estresse osmótico no neurônio e nas células da glia o que desencadearia a apoptose dessas.(ASHRAFIAN e DAVEY, 2001).

Em pacientes desidratados, quando submetidos à administração excessiva de soluções salinas podem apresentar um quadro neurológico compatível com mielinólise pontina (FREITAS e CARPENEDO, 2014; SNELL e BARTLEY, 2008).

A MPC e MEP são agrupadas dentro do contexto de Síndromes de Desmielinização Osmótica (SDO), e são caracterizadas pela dissolução das bainhas de mielina, poupando axônios nervosos. Como não há componente inflamatório, sugere-se que a morte celular seja ocasionada pela apoptose. Essa condição, na qual a perda da mielina ocorre sem qualquer infiltrado inflamatório óbvio, deve ser diferenciada da natureza inflamatória da esclerose múltipla (TANEJA et al., 1997; BRITO et al., 2006).

Objetivo deste estudo é relatar o caso de uma paciente com quadro de MPC após a rápida correção da hiponatremia. Este relato se faz de extrema importância, por se tratar de uma condição que não possui, ainda, tratamento eficaz, e sim prevenção. 


\section{RELATO DE CASO}

Mulher, 57 anos de idade, previamente hígida, natural e proveniente de Monte Azul-MG inicia um quadro de vômitos persistentes, agitação noturna, fadiga e epigastralgia, referidos durante um período de sete dias. Fez uso em um primeiro momento de ondansetrona, meclizina, pantoprazol, Cefalium ${ }^{\circledR}$ (mesilato de dihidroergtamina + paracetamol + cafeína + cloridrato de metoclopramida) e Vertizine $D^{\circledR}$ (mesilato de dihidroergocristina + dicloridrato de flunarizina), para controle dos sintomas. Após iniciar o uso dessas medicações, apresentou rebaixamento do nível de consciência, letargia, sonolência excessiva, fraqueza muscular e afasia. Atendida em hospital de referência da cidade próxima, foi constatado sódio sérico de 103 $\mathrm{mEq} / \mathrm{L}$, valor de referência $(\mathrm{VR})(135-145 \mathrm{mEq} / \mathrm{L})$ e potássio $(2,5 \mathrm{mEq} / \mathrm{L}, \mathrm{VR} 3,5-5,5 \mathrm{mEq} / \mathrm{L})$ iniciada correção dos distúrbio hidroeletrolítico no centro de tratamento intensivo (CTI) deste serviço. O resultado da Tomografia Computadorizada de Crânio (TC) não acusou alterações relevantes durante essa admissão.

A paciente apresentou uma elevação de $21 \mathrm{mEq} / \mathrm{L}$ em sua natremia durante as primeiras 24 horas (124 $\mathrm{mEq} / \mathrm{L}$ ). Após dois dias do início da correção do sódio, a paciente apresentou melhora significativa do quadro clínico, com retorno da fala e melhora do nível de consciência. Apresentou quadro febril, com resultado de exame de urocultura evidenciando processo infeccioso do trato urinário (ITU) que foi tratada com Ciprofloxacino.

Após 6 dias, com novo rebaixamento de consciência (Pontuação na Escala de Coma de Glasgow=10), com tetraparesia flácida e torpor, foi realizada Ressonância Magnética (RM) do Crânio que não revelou

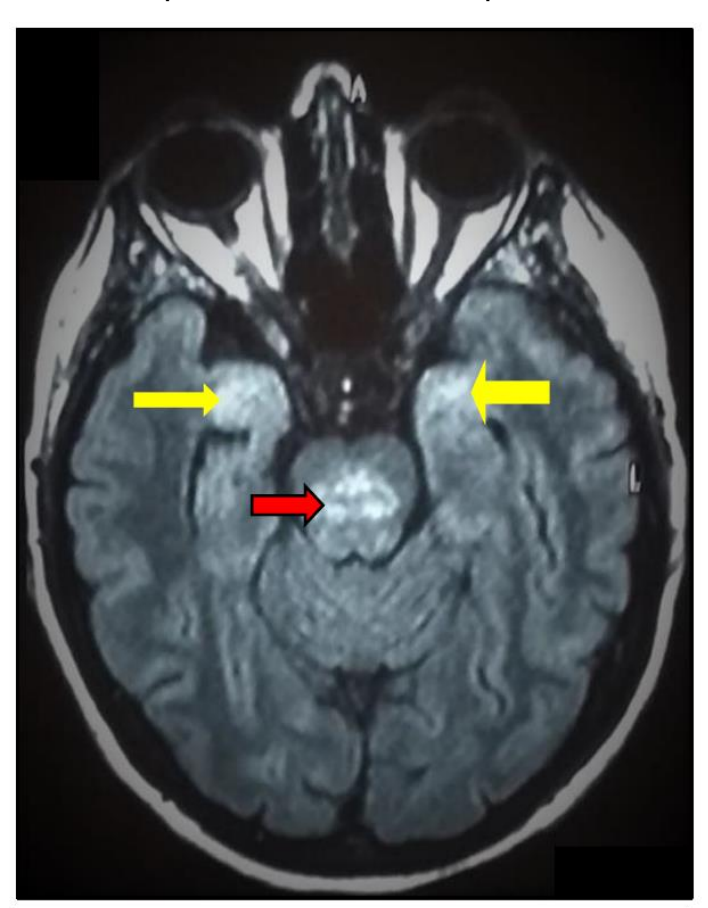

Figura 1: Imagem de Ressonância Magnética de Crânio (corte axial, sequência FLAIR). Setas amarelas revelam lesões hiperintensas nas amígdalas bilateralmente e seta vermelha hiperintensidadeem transição pontomesencefálica. Quadro compatível com mielinólisepontina central.

Fonte: imagem cedida pela paciente alterações relevantes. (Figura 1) Após alguns dias, a paciente revelava-se hemodinamicamente estável, porém com persistência da afasia e tetraparesia, sem comunicação externa. Pela persistência dos déficits neurológicos foi realizada nova RM: hiperintensidade nas sequências ponderadas em T2 e Flair na porção central da ponte, nos núcleos da base (núcleos lentiforme e caudado), no córtex dos giros pré-centrais, hemisférios cerebelares e amígdalas, sugestivas de mielinólise pontina.

Permaneceu internada no CTI deste serviço por dois meses para tratamento de suporte. Evoluiu neste período com insuficiência respiratória necessitando de intubação orotraqueal e posterior traqueostomia. No CTI, apresentou novo episódio de ITU associada à pneumonia aspirativa que foi tratado com Cefepime e Clindamicina.

Após dois meses, recebeu alta do CTI traqueostomizada, com tetraparesia espástica, paralisia pseudobulbar e consciência preservada. Recebeu alta hospitalar após 5 meses de internação, sem a traqueostomia e encaminhada à fisioterapia, fonoaudiologia, fisiatria e manutenção do acompanhamento neurológico. Em uso de Ranitidina, Heparina subcutânea, Metoprolol, Fenitoína e Clonidina.

Após 6 meses da alta hospitalar, a paciente em consulta de controle apresentava-se estável hemodinamicamente, alerta, respirando em ar ambiente sem traqueostomia, diurese e evacuações preservadas. Para locomoção necessitava do uso de cadeira de rodas, com manutenção adequada de postura, tetraparesia com força motora $4 / 5$. Seu exame neurológico revelava anosmia e alterações de sensibilidade. 
Apresentava episódios esporádicos de espasticidade durante o dia, que foram controladas após aplicação de toxina botulínica (realizada de 4 em 4 meses). Observou-se recuperação parcial da fala com disartria, dificuldade de deglutição, alimentando-se com alimentos pastosos. Em uso, na ocasião do relato, de Metoprolol 50mg 2x/dia, Fenitoina 100mg 2x/dia, Losartana 50mg 2x/dia, Sertralina 50mg 1x/dia, Baclofeno $20 \mathrm{mg} 2 x /$ dia, Zolpidem $10 \mathrm{mg}$ à noite e Alprazolam $1 \mathrm{mg}$. A paciente permanece em acompanhamento com neurologista, fonoaudiólogo, fisiatra e fisioterapeuta.

\section{DISCUSSÃO}

A fisiopatologia da mielinólise pontina é caracterizada por um quadro de desmielinização osmótica que pode acarretar diferentes déficits neurológicos. As células predominantemente atingidas são os oligodendrócitos (BRITO et al., 2006). Teoricamente vários fatores poderiam explicar essa seletividade no processo de lesão desse grupo celular:

-Distribuição linear dos neurônios e células da glia na estrutura da ponte que poderia limitar a capacidade de transporte/estoque de glicose e turgescência.

-Elevada necessidade metabólica e uma perfusão sanguínea menos efetiva.

-Características da adaptação das células da glia e neurônios durante o processo de hiponatremia.

-Elevada probabilidade de ocorrência de lesão secundária ao edema vasogênico pela proximidade dos núcleos localizados na ponte.

À medida que a água flui livremente através da barreira hematoencefálica e das membranas celulares durante o processo de hiponatremia acarreta a entrada de água nas células cerebrais e o consequente edema cerebral. Na tentativa de diminuir este edema, as células expulsão partículas osmoticamente ativas (como a taurina, mioinositol e o glitamato) para reduzir o acúmulo de água intracelular. Estes se perdem ao longo de um dia tornando a célula isotônica em relação ao fluido extracelular e com isso mantendo o seu volume (BROWN, 2000; RUZEKA et al., 2004; MARTIN, 2004).

Durante a correção de uma hiponatremia, se a taxa de aumento da tonicidade é mais rápida do que a taxa de formação e/ou transporte dos osmoles orgânicos para as células, estas irão de desidratar, o que culminará no processo de apoptose. Talvez seja aqui que o estado nutricional alterado do paciente desempenhe seu papel, reduzindo a capacidade de regenerar osmoles orgânicos, o que demonstra a importância destes para a proteção do encéfalo em mudanças rápidas na osmolalidade sérica (RUZEKA et al., 2004; MARTIN , 2004).

Estudos realizados em animais revelaram que níveis cerebrais de fosfocreatina, creatina, mioinositol, glicerofosforil colina, taurina, glutamato e glitamina se reduzem nas 24 horas iniciais após o iniciado a hiponatremia. Entretanto, de forma independente do processo de correção do íon sódio, essas substâncias tendem a se acumular nos tecidos cerebrais. Visto essa informação, pode-se inferir que por isso níveis de natremia adequados podem não excluir o diagnóstico de SDO.

Quanto às manifestações clínicas estas se apresentam de maneira bifásica. Inicialmente os pacientes apresentam-se com um quadro clínico de encefalopatia ou convulsões, secundárias à hiponatremia, posteriormente com melhora clínica evidente proporcionalmente à correção da hiponatremia. Entre 2 a 8 dias da correção da hiponatremia, os pacientes usualmente apresentam paralisia pseudobulbar, tetraparesia com arreflexia e hipotonicidade muscular. A hipotonia inicial, posteriormente transforma-se em espasticidade. Nesta fase, os pacientes podem cursar com alterações do nível de consciência, Síndrome do encarceramento que também é denominada Locked-in Syndrome, coma ou óbito. Ocorre ainda ataxia, disartria, labilidade emocional, acinesia, distonia, coreoatetose, mioclonias, rigidez, alterações oculomotoras e tremor. A consciência é preservada na maioria das vezes (BENTO, 2013; ADAMS et al., 2009; ASHRAFIAN, 2001 et. al.). Muitos dos sinais e sintomas supracitados estiveram presentes nas manifestações clínicas da paciente em questão.

Nota-se que alguns pacientes desenvolvem MPC e MEP com concentrações plasmáticas do íon sódio mais elevadas e também com menor velocidade da correção deste. Sabe-se que o principal fator que desencadeia a MPC e MEP é a rápida correção do sódio, principalmente quando essa correção é superior a 
$10 \mathrm{mEq} / \mathrm{L}$ dia em pacientes com níveis séricos de sódio inferiores ou iguais a $120 \mathrm{mEq} / \mathrm{L}$. Alguns fatores de risco são conhecidos tais como: sepse, hemodiálise, neoplasias, pós-operatório de procedimentos urológicos, ginecológicos e da neurocirúrgicos (cirurgia de hipófise), doenças hepáticas graves, Diabetes insipidus, etilismo crônico e desnutrição (MARTIN, 2004, FREITAS e CARPENEDO, 2014; TANEJA et al., 1997).

A causa base para a hiponatremia do caso relatado não foi identificada, no entanto, os vômitos podem ser apontados como causa e/ou consequências da alteração natrêmica (ROCHA, 2011). As medicações utilizadas em um primeiro momento pela paciente não apresentam como efeitos adversos a hiponatremia. $O$ quadro neurológico posterior é justificado e previsto pela reposição abrupta da natremia. O caso descrito corrobora a reposição lenta e limitada de sódio em quadros de hiponatremia, sendo esta a principal medida profilática para esta patologia (TANEJA et al.,1997). A correção rápida da hiponatremia ocorreu no caso relatado, aumentando-se o sódio sérico além do previsto e orientado pela literatura.

O quadro neurológico poderia também ser justificado por um acidente vascular encefálico isquêmico $(\mathrm{AVCl})$, onde a paciente apresentaria uma clínica de déficit neurológico agudo, referente à área lesada. Comumente com alterações neurológicas em apenas um hemicorpo, relacionada diretamente ao hemisfério encefálico contrário, associado à alteração de mímica facial, afasia, disartria e uma história pregressa de fatores de risco, que possibilite a formação de trombos e êmbolos. A esclerose múltipla quando acomete $o$ tronco encefálico por apresentar sintomas semelhantes aqueles presentes na MPC e MEP e por isso deve ser aventada como um diagnóstico diferencial. No exame de ressonância magnética do crânio, a esclerose múltipla pode apresentar o sinal dos "Dedos de Dawson" no corpo caloso, os quais não estão presentes na MPC e MEP. (ADAMS et al., 2009).

Quanto ao diagnóstico radiológico, sabe-se que as imagens sugestivas da MPC podem não serem evidentes na TC de crânio ou na RM inicial, principalmente com um período inferior a uma semana de evolução. A RM de crânio, principalmente as sequências T2 e Flair são úteis para o diagnóstico (BRITO et al., 2006; RUZEKA et al., 2004).

Nas sequências $T 1$, as lesões apresentam-se hipointensas e simétricas, preservando as regiões periféricas pontinas. Nas sequências T2 e FLAIR, as lesões se revelam hiperintensas. Um sinal que pode estar presente nesse grupo de pacientes é o sinal da "asa de morcego" na base da ponte (BRITO et al., 2006; RUZEKA et al., 2004).

Não há estudos suficientes até o presente momento que definam um tratamento específico para a MPC e MEP. Existem na literatura médica estudos que citam a utilização de corticoide, imunoglobulina humana e hormônio liberador de tireoglobulina para o tratamento desses pacientes, entretanto ainda sem adequada evidência científica. (BRITO et al., 2006; JUMO et al., 2012).

O tratamento é uma abordagem de suporte, em muitos casos apenas cuidados paliativos (RUZEKA et al., 2004; JUMO et al.,2012). O caso em questão nota-se que a paciente apresentou uma melhora expressiva das funções cognitivas e motoras com o tratamento das complicações clínicas da MPC. A reposição natrêmica, à qual a paciente foi submetida, foi de $21 \mathrm{mEq}$ em 24 horas, não seguindo as orientações atuais: reposição máxima de 10 a $12 \mathrm{mEq}$ em 24 horas, sendo $3 \mathrm{mEq}$ nas primeiras 3 horas com solução salina hipertônica, levando em consideração a quantidade de água corporal e a variação de sódio esperada (FREITAS e CARPENEDO, 2014). Estudos indicam melhora da qualidade de vida com o uso de toxina botulínica em condições que cursam com espasticidade (SEGURA et al., 2005), demonstrada claramente na evolução da paciente.

O prognóstico da MPC é reservado. Um estudo envolvendo 34 casos de MPC/MEP concluiu que a mortalidade está relacionada com a ocorrência de complicações tais como pneumonia de aspiração, infecções do aparelho urinário, sepse, trombose venosa profunda e tromboembolismo pulmonar (ASHRAFIAN, 2001; MENGER e JARG, 1999). Contudo, os pacientes podem apresentar desde recuperação total, sem sequelas, ou mesmo sequelas neurológicas irreversíveis (BRITO et al.,2006).

Dessa forma, nenhum tratamento está estabelecido além do tratamento de suporte. É importante, uma vez que o paciente melhore que este seja inserido em um processo de reabilitação com uma equipe 
multidisciplinar, que inclua reabilitação física, terapia de deglutição, linguagem, entre outras, para que sejam minimizadas as sequelas e para sua reintrodução na sociedade, obtendo uma melhor qualidade de vida (RUZEKA et al., 2004; JUMO et al.,2012).

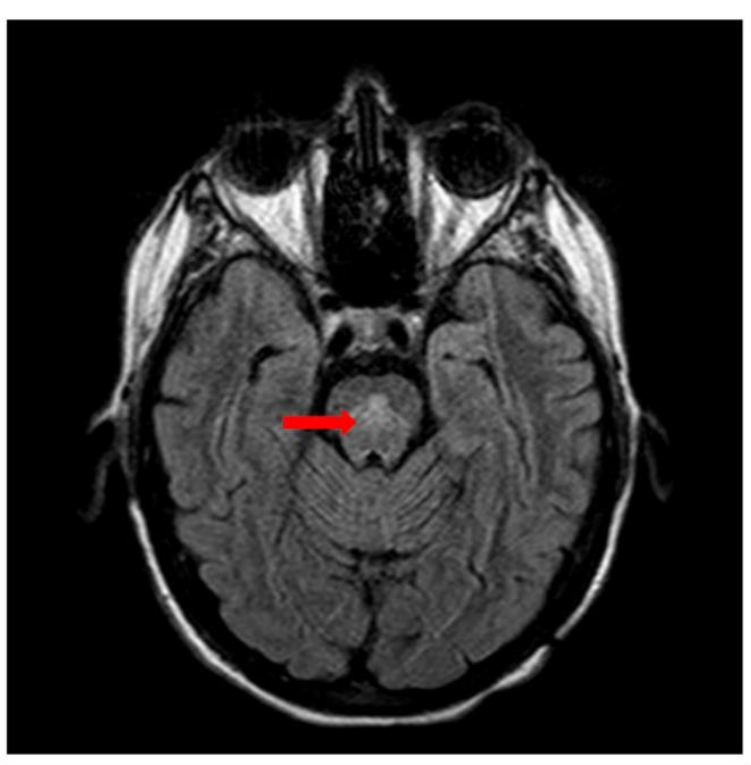

Figura 2: Imagem de Ressonância Magnética de Crânio (corte axial, sequência FLAIR). Seta vermelha apresentando regressão parcial da lesão hiperintensa em transição ponto-mesencefálica. Fonte: imagem cedida pela paciente

A paciente foi submetida a um tratamento de suporte preconizado, tendo em vista a ausência de tratamento curativo para esta condição (BRITO et al., 2006). A mesma vem sendo submetida a sessões de fisioterapia motora e respiratória, acompanhamento com fonoaudiólogo e fisiatra, demonstrando uma evolução progressiva das capacidades motoras e cognitivas, que, na maioria das vezes, não é esperada na evolução desse quadro (MENGER e JARG, 1999). Após 10 meses do quadro inicial, a paciente apresenta uma melhora significativa em sua qualidade de vida associada com melhora das alterações estruturais nos últimos exames de imagens (RM), que evidenciaram regressão de áreas acometidas. (Figura 2)

A correção da hiponatremia deve sempre seguir as orientações estabelecidas na literatura vigente, respeitando os limites diários de elevação do sódio sérico, para se evitar o desenvolvimento de mielinólise pontina. Salienta-se a importância da associação entre exame clínico específico e imagens de RM ponderadas nas sequências T2 e Flair para o diagnóstico, principalmente quando solicitada em tempo adequado. O relato evidencia a necessidade do suporte e acompanhamento multidisciplinar a uma paciente acometida por mielinólise pontina, demonstrando que apesar de ser uma doença sem tratamento específico e curativo, há possibilidade de resposta à estimulação motora e cognitiva. Sendo assim, a principal abordagem sempre será a prevenção primária, impedindo o desenvolvimento do quadro em questão.

\section{REFERÊNCIAS}

1. BENTO S, AUGUSTO A, JACINTO LJ. Mielinólise pontina: a propósito de um caso clínico. Revista as Sociedade Portuguesa de Medicina Física e de Reabilitação, 2013; vol.23.

2. ADAMS RD, VICTOR M, MANCALL EL. Central pontine myelinosis: a hitherto undescribed disease occurring in alcoholic and malnourished patients. Archives Neurology Psychiatry, 1959; 81:154-6.

3. BROWN WD. Osmotic demyelination disorders: central pontine and extrapontine myelinolysis. Current Opinion in Neurology, 2000; 13:691- 697 .

4. ADAMS RD, VICTOR M, ROPPER AH. The acquired metabolic disorders of the nervous system-central pontine myelinolysis. Principles of Neurology. 9th edition. New York: McGraw Hill, 2009; 1097-9p.

5. ASHRAFIAN H, DAVEY P. A review of the causes of central pontine myelinosis: yet another apoptotic illness? European Journal Neurology, 2001; 8:103-109.

6. FREITAS BA, CARPENEDO CM. Mielinólise pontina em paciente etilista. Revista da AMRIGS, 2014; 58: 268-271.

7. TANEJA K, SABHARAL RK, TANEJA A et al.Central pontine myelinolysis in a normonatremic child. Indian Pediatrics, 1997; 34:153156.

8. SNELL DM, BARTLEY C. Osmotic demyelination syndrome following rapid correction of hyponatraemia. Anaesthesia, 2008; 63: 9295.

9. BRITO AR, VASCONCELOS MM, JÚNIOR LCHC et al. Central Pontine and extrapontine myelinolysis: report of a case with a tragic outcome. Jornal de Pediatria, 2006; 82 (2): 157-160.

10. RUZEKA KA, CAMPEAUA NG, MILLER GM. Early diagnosis of central pontine myelinolysis with Diffusion-Weighted Imaging. American Journal of Neuroradiology, 2004;25: 210-213.

11. MENGER H, JARG T. Outcome of central pontine and extrapontine myelinolysis. Journal Neurology, $1999 ; 246: 700-705$.

12. JUMO ME, CASTRO MHA, LAGE MA et al. Osmotic demyelination syndrome: report of a case with favorable outcome. Radiologia Brasileira, 2012; 45:61-62.

13. SEGURA DCA, ADAMCHUK CC, NASCIMENTO FC et al. A utilização da toxina botulínica associada à fisioterapia para o controle da espasticidade. Arquivos de Ciências da Saúde da Unipar, 2005; 9(3): 217-222.

14. ROCHA PN. Hiponatremia: conceitos básicos e abordagem prática. Jornal Brasileiro de Nefrolologia, $2011 ; 33$ (2):248-260.

15. MARTIN RJ. Central pontine and extrapontine myelinolysis: the osmotic demyelination syndromes. Journal of Neurology Neurosurgery and Psychiatry, 2004;75:22-28. 